\section{Cahiers de Narratologie}

Analyse et théorie narratives

$10.2 \mid 2001$

La voix narrative

\title{
La voix autobiographique
}

\section{Susan Marson}

\section{OpenEdition}

Journals

Édition électronique

URL : http://journals.openedition.org/narratologie/10225

DOI : 10.4000/narratologie.10225

ISSN : 1765-307X

Éditeur

LIRCES

Édition imprimée

Date de publication : 1 janvier 2001

Pagination : 233-241

ISBN : 2914561032

ISSN : 0993-8516

Référence électronique

Susan Marson, «La voix autobiographique », Cahiers de Narratologie [En ligne], 10.2 | 2001, mis en ligne le 01 janvier 2001, consulté le 11 juin 2020. URL : http://journals.openedition.org/narratologie/10225 : DOI : https://doi.org/10.4000/narratologie. 10225 


\title{
LA VOIX AUTOBIOGRAPHIQUE
}

\author{
Susan MARSON \\ University of London
}

Tout ceci doit être considéré comme étant dit par un personnage de roman'.

Qui suis-je ? Si par exception je m'en rapportais à un adage : en effet pourquoi tout ne reviendrait-il pas à savoir qui je 'hante' ??

L'autobiographie se définit, en principe, par l'unité du sujet : auteur, narrateur et personnage ne font qu'un. L'unité linguistique du discours à la première personne recouvre cependant trois instances dont la fonction (écrire, narrer, agir), dans chaque cas, est bien différente. Qui parle, donc, dans l'autobiographie ? Il n'y a personne qui puisse parler, dans le texte écrit, si ce n'est cette instance qu'on appelle le narrateur. Or l'instance narrative est fictive de nature, car le propre de la narration, c'est de faire entendre une voix dont l'origine, comme la sonorité, sont absentes. L'autobiographie est en ce sens contrainte d'emprunter une voix fictive pour faire entendre le vécu réel de l'écrivain, développant des structures textuelles complexes afin de rattacher cette voix au corps de l'auteur. Mon propos d'aujourd'hui sera de suggérer que le texte autobiographique - dont je voudrais énumérer dix aspects formels en particulier - travaille à relier la voix narrative au monde réel, de telle sorte qu'il y a bien lieu de parler d'une voix proprement autobiographique, même s'il s'agit d'une voix désincarnée, fabuleuse, fantômale.

1 Roland BARTHES, Roland Barthes, Seuil, coll. Les écrivains de toujours, p. 5.

2 André BRETON, Nadja, Gallimard Folio, p. 9. 
$\S 1$. L'autobiographie est d'abord, de toute évidence, un récit. Nous le verrons, elle n'est pas entièrement rétrospective, et sa prose se fait souvent fragmentaire et poétique, comme en témoignent les textes de Michel Leiris, ou de Jacques Roubaud. De fait, il y a un lien étroit entre poésie et autobiographie ; ce sont deux genres pour lesquels la forme écrite est de première importance. L'autobiographie est donc tout d'abord récit écrit; elle a pour but non seulement de raconter une histoire, mais, nous le verrons, de créer un livre. Ainsi Rousseau, dans le passage célèbre du début des Confessions : "Que la trompette du Jugement dernier sonne quand elle voudra, je viendrai, ce livre à la main [...]. Je dirai hautement : "Voilà ce que j'ai fait, ce que j'ai pensé, ce que je fus. [...]" »33. La voix, dans l'autobiographie, est une voix écrite : c'est le livre, dirait-on, qui parle.

$\S 2$. L'autobiographie est donc récit, mais elle est aussi discours au sens de Benveniste : elle se sert de la première personne et des temps du présent, se référant à l'ici-etmaintenant de la deixis et invoquant le lecteur ${ }^{4}$. "Voici le seul portrait d'homme - nous dit encore Rousseau - peint exactement d'après nature et dans toute sa vérité, qui existe et qui probablement existera jamais. Qui que vous soyez, que ma destinée ou ma confiance ont fait l'arbitre du sort de ce cahier, je vous conjure [...]». Cet usage de formes linguistiques discursives, même de manière intermittente dans le texte, a pour effet de créer un lien étroit entre passé et présent, entre les événements à raconter et leur mise en récit. Ainsi chez Rousseau encore, lorsqu'il raconte un souvenir d'enfance : "Je sens en écrivant ceci que mon pouls s'élève encore ; ces moments me seront toujours présents quand je vivrais cent mille ans. $»^{5}$

3 Jean-Jacques ROUSSEAU, Les Confessions, t. 1, Livre premier, Livre de poche, pp. 5-6.

4 Voir Emile BENVENISTE, Problèmes de linguistique générale, t. 1, Gallimard, 1966.

5 Idem, p. 29. 
§3. En effet, à l'instar de Rousseau, l'autobiographie s'ouvre très souvent sur une mise en scène de l'écrivain s'adressant au lecteur pour s'expliquer sur le texte qui va suivre: le discours autobiographique devient commentaire, et le texte se fait doubler de son propre discours critique ou métadiscours, fournissant au lecteur le mode d'emploi du texte tenu devant ses yeux. Ainsi le début de cet étrange petit texte de Pierre Pachet, Autobiographie de mon père: "Sans doute est-il nécessaire que je m'explique, moi Pierre Pachet, sur le texte étrange qu'on va lire et pour lequel j'ai tenu la plume. Quel est le sens de ce projet, et comment l'ai-je réalisé ? »6. Stendhal, dans Vie de Henry Brulard, commente même l'usage du commentaire : "Aurai-je le courage d'écrire ces confessions d'une façon intelligible ? Il faut narrer, et j'écris des considérations sur des événements bien petits mais qui précisément à cause de leur taille microscopique ont besoin d'être contés très distinctement. Quelle patience il vous faudra, ô mon lecteur ! »?

$\S 4$. Cet usage du commentaire travaille à brouiller les limites du texte pour s'ouvrir sur le monde réel. En donnant le mode d'emploi du livre, le métadiscours qui se mélange au récit autobiographique s'adresse, en principe, à un narrataire ; mais c'est un lecteur implicite qui se confond avec le lecteur réel. De fait, le discours confessionnel s'adresse toujours, en puissance, à un lecteur ayant droit de réponse et de jugement. «Faire un livre qui soit un acte, écrit Michel Leiris de son Âge d'homme, [...] Acte par rapport à autrui, puisqu'il était évident qu'en dépit de mes précautions oratoires la façon dont je serais regardé par les autres ne serait plus ce qu'elle était

6 Pierre PACHET, Autobiographie de mon père [Belin, 1987], Autrement, 1994, p. 5. Voir aussi le commentaire de J.-B. Pontalis, 'Derniers, premiers mots' dans L'Autobiographie, Vièmes rencontres psychanalytiques d'Aix-en-Provence, ed. M. Neyraut, Les Belles Lettres, 1990.

7 STENDhal, Vie de Henry Brulard, préface de Béatrice Didier, Gallimard Folio, p. 43. 
avant publication de cette confession. $»^{8}$ " $[\mathrm{A}] \mathrm{u}$ fond de toute confession, écrit encore Leiris, il y a désir d'être absous. ${ }^{9}$ Désir d'absolution, mais désir parfois aussi de réglement de comptes, lorsqu'un livre d'Hervé Guibert s'adresse "à l'ami qui ne m'a pas sauvé la vie », ou que Serge Doubrovsky raconte dans Le Livre brisé : « justement, si j'écris, c'est pour tuer une femme par livre $» .10$

$\S 5$. Le propre du discours autobiographique, c'est donc de s'adresser à un lecteur réel, en lui parlant du texte qu'il est en train de lire : la voix autobiographique nous parle d'écriture. C'est donc un discours paradoxalement autoréférentiel, dont l'objet n'est pas seulement le passé que raconte le récit, mais aussi le présent de l'écriture : peindre, dit Augustin, non ce que je fus, mais * ce que je suis, dans le temps même où je rédige... ${ }^{11}$. Cet usage du discours pour rendre vivant l'écriture est une des particularités, me semble-t-il, du texte autobiographique. Ainsi, par exemple, la mise en scène de l'acte d'écrire à la première page de La Bâtarde, de Violette Leduc :

Lecteur, mon lecteur, j'écrivais dehors sur la même pierre il y a un an. Mon papier quadrillé n'a pas changé, l'alignement des vignes est pareil au-dessous de la chevauchée des collines. Au troisième rang, c'est encore la buée de chaleur. Mes collines baignent dans leur auréole de douceur. Suis-je partie, suis-je revenue ? Vivre ne serait donc plus mourir sans répit avec les secondes...12

§6. Cependant, précisément parce qu'il s'agit d'un texte écrit, le discours autobiographique provient d'un sujet sans voix,

8 Michel LEIRIS, 'De la littérature considérée comme une tauromachie', L'Âge d'homme, Gallimard Folio, pp. 14-15.

${ }^{9}$ Idem, p. 13.

10 Voir Hervé GUIBERT, A l'ami qui ne m'a pas sauvé la vie, Gallimard Folio, 1990 ; Serge Doubrovsky, Le Livre brisé, Grasset 1989, Livre de poche, p. 60.

11 Augustin, Les Confessions, Livre Dixième, Ch. 3, trad. Joseph Trabucco, Flammarion, 1964, p. 204.

12 Violette LEDUC, La Bâtarde, Gallimard, 1964, p. 19. 
pour s'adresser à un lecteur absent. En effet, la voix narrative en autobiographie ne peut se définir en se servant des formes discursives qui rapprochent l'écrivain du lecteur; au contraire, le présent de l'écriture a besoin, précisément, des formes historiques qui semblent éloigner le sujet, comme si, d'un coup, personne ne parlait, ou que la voix ne provenait plus d'une présence vivante. "J'étais presque mort quand je vins au jour ", écrit Chateaubriand après s'être servi des archives de l'église pour citer sa date de naissance le 4 septembre $1768^{13}$. La formule est très proche de celle de Rousseau :

J'étais né presque mourant ; on espérait peu me conserver. J'apportai le germe d'une incommodité que les ans ont renforcée, et qui maintenant ne me donne quelquefois des relâches que pour me laisser souffrir plus cruellement d'une autre façon. ${ }^{14}$

Comme Louis Marin l'a montré dans La Voix excommuniée ${ }^{15}$, la voix autobiographique fait entendre un discours historique (je vins au jour, j'apportai le malheur), ancré dans le passé par l'usage de l'aoriste, de dates, de noms de lieux, ramené vers le présent par un «je » qui, ayant pris soin de laisser toujours des traces de son existence précaire, s'énonce dans l'ici-et-maintenant du lecteur. L'autobiographie se sert donc des tours du langage oral pour rendre présente l'écriture, tout en se servant de traces historiques écrites pour fixer la provenance de cette voix déjà disparue. Ainsi le passage de Violette Leduc de tout à l'heure, où elle invoquait la présence du lecteur pour assister à la scène de l'écriture, finit-il par une inscription de dates qui viennent définir aprèscoup ce moment de présence : «Lendemain matin, 8 heures du matin du 24 juin 1962. J'ai changé d'endroit, j'écris dans les bois à cause de la chaleur. »

13 Mémoires d'outre-tombe, t. 1, Livre premier, ch. 2, Gallimard Pléiade, p. 17.

14 ROUSSEAU, p. 9.

15 Louis MARIN, La Voix excommuniée : essais de mémoire, Galilée, 1981 , p. 22-28. 
§7. Si le langage qu'emploie l'autobiographie est donc hybride, il en va de même de sa structure narrative. En effet, le récit autobiographique n'est pas entièrement rétrospectif, car il raconte aussi l'histoire présente et continue de la narration même. Le plus souvent, l'autobiographie souligne cette duplicité narrative par l'usage intercalé de passages parfois fragmentaires, souvent datés, se rapprochant du journal, de l'autoportrait ou du carnet de notes. Ainsi Chateaubriand écrit-il toujours en tête des chapitres la date et le lieu de leur écriture, voire des révisions et différentes versions :

Livre Douzième, Chap. 6. Londres, d'avril à septembre 1822.

Livre Treizième, Chap. 1. Dieppe, 1836. Revu en décembre 1946.

Livre Quatorzième. Paris, 1937. Revu en décembre 1846.

Livre Quinzième. Paris, 1838. Revu le 22 février 1845.

Ces dates notées forment une narration intercalée, minime certes, qui s'ajoute au récit rétrospectif et qui est souvent d'un égal intérêt. Chez Stendhal, il semble souvent que l'autobiographie est à lire tant dans l'histoire de son enfance grenobloise que dans les émotions éprouvées à écrire, puis à ne pas pouvoir achever sa Vie de Henry Brulard. " Je trouve quelquefois beaucoup de plaisir à écrire, voilà tout », écrit Stendhal au début de son texte ; quatre cents pages plus tard, il parle toujours de plaisir, mais c'est d'une joie cette fois-ci qui empêche l'écriture: "Comment peindre le bonheur fou? [...] Ma foi je ne puis continuer, le sujet surpasse le disant $»^{16}$. Stendhal commentera encore cette interruption dans les marges du texte :

«1836, 26 mars, annonce du congé [...]. L'imagination vole ailleurs. Ce travail en est interrompu. L'ennui engourdit l'esprit, trop éprouvé de 1832 à 1836 , Omar. "17

«Le 26 mars 1836, à dix heures et demie, lettre très polie pour gékon [congé]. Depuis ce grand courant dans mes idées, je ne travaille plus. ler avril 1836. ${ }^{18}$

16 STENDHAL, Vie de Henry Brulard, pp. 31, 434.

17 Idem, p. 435, n. 3 [p. 499].

18 Ibid., p. 433, n. 2 [p. 499]. 
$\S 8$. La narration, dans l'autobiographie, s'inscrit donc comme processus ayant une durée propre, à l'encontre de la narration fictive instantanée. Dans un récit entièrement rétrospectif, le présent de la narration demeure hors du temps; ainsi pour Genette, parlant de Proust, l'acte de raconter est " instantané, sans dimension temporelle »; il est "extase, "durée d'un éclair", miraculeuse syncope, "minute affranchie de l'ordre du Temps" ", et le présent du narrateur est " un moment unique et sans progression ". ${ }^{19}$ Ce n'est pas le cas dans l'autobiographie, comme le remarque d'ailleurs en passant Philippe Lejeune, dans une note en bas de page de son Pacte autobiographique. Raconter, écrire, ces actes prennent du temps, et leur durée est soit mise en scène dans le récit même, soit indiquée par des dates de rédaction marquées à la fin du texte $^{20}$. De fait, pour Genette, La Recherche de Proust doit se lire comme fiction, et non pas comme autobiographie, précisément parce que le temps de l'écriture du livre ne s'inscrit pas dans le texte même. Marcel a beau arriver, à la dernière page, au moment où il deviendra écrivain, l'acte réel d'écrire a lieu en dehors du livre, et le narrateur demeure donc instance fictive, voix pure. Or, continue Genette, la pratique courante de la narration autobiographique procéderait tout autrement, conduisant le héros au point où l'attend le narrateur, à la dernière page du texte, où ils écrivent ensemble, d'une même main, le mot « fin $» 21$.

\$. Ce que souligne Genette, c'est que dans l'autobiographie, la durée de la narration devient durée d'écriture liée au temps réel de la production du manuscrit ; cette durée de l'écriture est un composant essentiel du temps du récit autobiographique. En effet, l'autobiographe ne peut rendre compte de sa vie sans traiter aussi de l'activité principale qui la définit : l'acte d'écrire. Plus : il ne peut pas rassembler le temps

19 Gérard GENETTE, 'Discours du récit', Figures III, Seuil, 1972, p. 234 et sq.

20 Philippe LEJEUnE, Le Pacte autobiographique, Seuil, p. 199, n. 3 .

21 Gérard GENETTE, op. cit., pp. 233, 236. 
d'une vie dans l'espace du texte sans faire face à la durée de ce processus même. Écrire et vivre deviennent si fortement imbriqués qu'on ne peut parler de l'un sans faire référence à l'autre; et l'on ne peut mettre fin à l'écrit sans menacer la vie du même mouvement. Ainsi Chateaubriand, à la fin de ses mémoires :

En traçant ces derniers mots, ce 16 novembre 1841 , ma fenêtre, qui donne à l'ouest sur les jardins des Missionsétrangères, est ouverte : il est six heures du matin; j'aperçois la lune pâle et élargie ; elle s'abaisse sur la flèche des Invalides à peine révélée par le premier rayon doré de l'Orient : on dirait que l'ancien monde finit, et que le nouveau commence. Je vois les reflets d'une aurore dont je ne verrai pas se lever le soleil. Il ne me reste qu'à m'asseoir au bord de ma fosse : après quoi je descendrai hardiment, le crucifix à la main, dans l'éternité.

\section{FIN DES MÉMOIRES.}

Cette mise en scène correspond très bien à la situation décrite par Genette : héros et narrateur se rencontrent pour finir ensemble, et la vie, et son récit. Mais il s'agit surtout, ici, d'une très belle fiction. Car si héros et narrateur s'assoient ensemble au bord de la fosse, ils devront attendre longtemps pour que l'écrivain, lui, vienne les rejoindre. Nous l'avons déjà vu, Chateaubriand est encore à corriger et à revoir son manuscrit en 1846, cinq années plus tard. Son texte nous raconte une histoire qui finit comme il sied, mais il nous montre aussi le travail d'écriture, de correction et de composition du manuscrit nécessaire pour créer un livre.

$\S 10$. De fait, l'histoire, dans l'autobiographie, n'est jamais achevée : l'écrivain ne peut raconter toute sa vie ; son texte s'interrompra toujours avant le moment de sa mort. La fin du texte cependant nous est connue dès le début du récit, car le livre autobiographique forme sa propre conclusion ${ }^{22}$. La fin

22 Voir H. Porter ABBOTT, 'Autobiography, Autography, Fiction : Groundwork for a Taxonomy of Textual Categories', New Literary History, n. 19, 1988, p. 597. 
du texte, son but et sa destination, c'est le livre même, cet objet réel entre les mains du lecteur.

L'autobiographie a donc ceci de particulier, que le temps de la narration s'ouvre sur le temps de l'écriture en tant qu'acte réel, ou pour le dire autrement, que la voix narrative se rattache à la main écrivante pour esquisser une figure du corps de l'écrivain (de telle sorte que le corps, me semble-t-il, est bien 'pertinent', pour reprendre le mot de Mieke Bal ce matin). L'acte autobiographique, c'est ce qui produit le livre que nous tenons, nous, lecteurs, devant nos yeux, comme trace de ce corps absent ; ouvrant le livre, tournant les pages, suivant les lignes d'encre noire des phrases, c'est comme si nous entendions une voix - quelle voix ? Et de qui ? C'est une voix désincarnée, muette, qui n'est plus que l'écho lointain des traces de l'écrit ; fantômale, elle n'est pas pour autant entièrement fictive. Elle est, certes, une fabrication, une abstraction sans matérialité tangible ; elle se sert même de son état désincarné pour nous raconter des fables, telle cette voix chez Chateaubriand qui veut nous parler d'au-delà de la mort. Cette voix fabuleuse revêt cependant une double vérité, car en faisant son propre commentaire elle nous montre les procédés réels de sa fabrication dans l'écriture. Même lorsqu'elle se peint en voix d'outre-tombe, elle ne nous dit que la vérité : la voix autobiographique est, en effet, une voix de fantôme. Le livre de Pierre Pachet, écrit pour la plupart dans la voix de son père mort, nous le montre de façon très claire : «[...] ce que j'ai retranscrit dans cette "Autobiographie" : une parole qui s'écrivait, se reprenait, ne parlant à personne, à la recherche d'une certaine façon de s'exprimer, d'une vérité qui lui échappait... ». ${ }^{23}$ Nous lisons les mots sur la page et nous l'entendons, cette voix de mort, voilà qu'elle nous fait voir aussi la main tenant la plume, le geste d'écrire et de biffer, la figure sombre de l'écrivain au travail. Une fiction, tout cela ? Non, pas du tout, cette histoire d'écriture a bien eu lieu : ce livre que je tiens ici à la main en est la preuve.

23 Op. cit., p. 7. 